\title{
DO THE OH/IR STARS WITHIN 100 PC OF THE GALACTIC CENTER BELONG TO THE DISK POPULATION?
}

\author{
A. E. Whitford \\ Lick Observatory, University of California \\ Santa Cruz, California 95064 USA
}

The $135 \mathrm{OH} / \mathrm{IR}$ stars within $0.8 \mathrm{deg}(\sim 100 \mathrm{pc})$ of the Galactic Center (GC) identified in a search with the VLA (Lindqvist et al. 1991) are in rapid rotation about the center with a regression line showing a velocity gradient of $1.2 \pm 0.1 \mathrm{~km} \mathrm{~s}^{-1}$, equivalent to $178 \mathrm{~km} \mathrm{~s}^{-1} \mathrm{deg}^{-1}$ for $R_{o}=8.5 \mathrm{kpc}$ (Lindqvist et al. 1992). This is of the same order as the inward extrapolation of the Galactic rotation curve derived from the terminal velocities of $\mathrm{H}_{\mathrm{I}}$ for $l<2$ deg (Burton and Gordon 1978) and the general trend of the velocities of the CO clouds in the equatorial disk mapped by Dame et al. (1987) (Fig. 1b). These OH/IR stars are located along the ridge of highest projected $\mathrm{CO}$ density. This spatial location and the flat configuration argue for assignment to the disk population, a possibility suggested by Feast (1989). The VLA objects are not in general supergiants (Blommaert et al. 1992). On a K-band map of an area $1 \times 2 \mathrm{deg}$ around the GC, Catchpole et al. (1990) found a number of stars brighter than $\left(m_{K}\right)_{0}=5$. On the assumption that $\mathrm{BC}_{\mathrm{K}}=3.3$, (Frogel and Whitford 1987), and $\mathrm{R}_{\mathrm{o}}=8.5 \mathrm{kpc}, \mathrm{M}_{\mathrm{bol}}<-6.3$, i.e., in the supergiant range.

Lindqvist et al. (1992) interpreted the greater scale height and velocity dispersion of the less luminous fraction of the VLA sample (judged by smaller average shell expansion velocity $V_{\text {exp }}$ ) as an age difference; the longer life of these stars allows time for a disk heating process (Wielen et al. 1992) to operate. This suggests an age spread like that in the classical disk population.

In order to compare the kinematics of $\mathrm{OH} / \mathrm{IR}$ stars near the GC with other OH/IR stars in the inner Galaxy, a sample was taken from the Effelsberg-Parkes survey (te Lintel Hekkert et al.

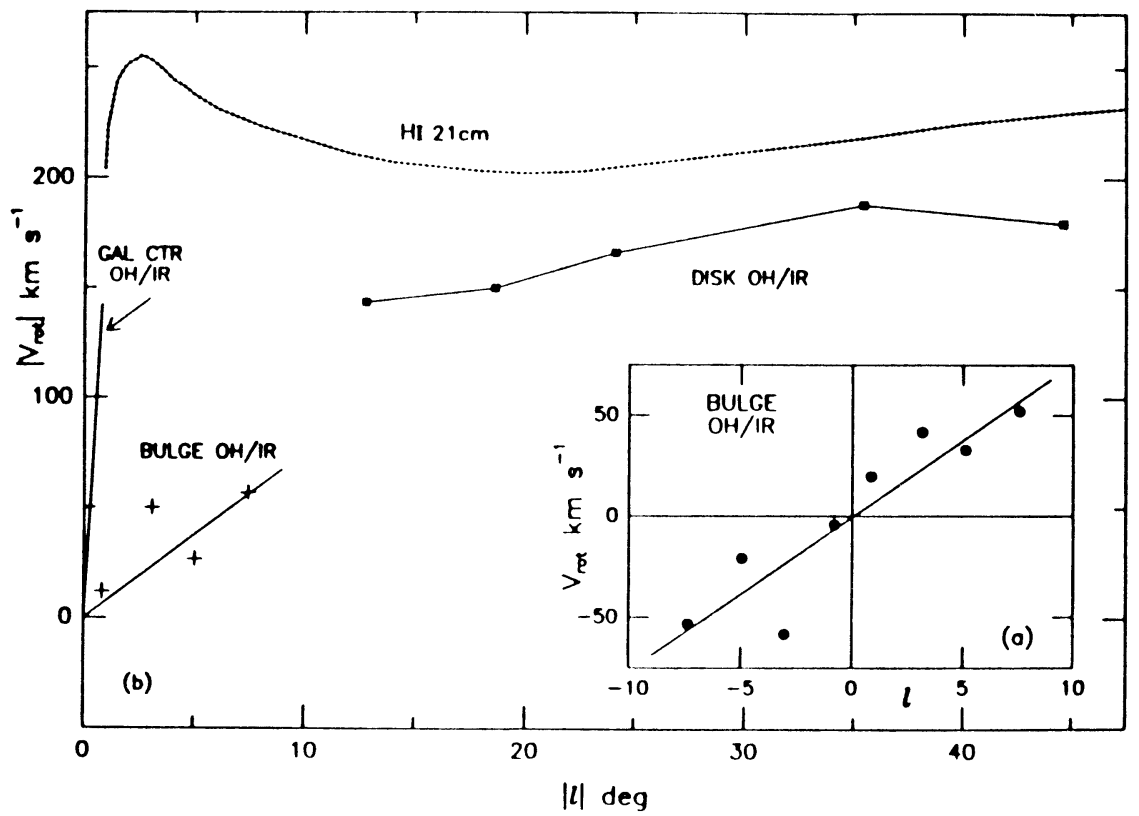

Figure 1. (a) Group means for bulge OH/IR stars, $|l|<10$, and regression line. (b) Regression lines showing rotation velocity gradient for OH/IR stars in Galactic center and bulge,and group means for disk $\mathrm{OH} / \mathrm{IR}$ stars. Dotted line: Galactic rotation curve from $\mathrm{H}_{\mathrm{I}}$ clouds. 
1991). Objects with thick circumstellar shells having IRAS colors IIIb were selected. Van der Veen and Habing (1990) showed that for this class of objects the survey is very nearly complete to the distance of the GC.

The 59 stars in the sample within the range $10<|l|<50$ show Galactocentric velocities, $\mathrm{V}_{\mathrm{rot}}$, that mark them as disk objects. Group means are shown in Figure $1 \mathrm{~b}$. The points fall below the terminal velocities for $\mathrm{H}_{\mathrm{I}}$ because the stars are in general not at the tangent point, and because of the asymmetric drift found by Lewis and Freeman (1989).

The $80 \mathrm{OH} / \mathrm{IR}$ objects in the selected sample with $|l|<10$ define the bulge kinematics. Group means for 8 bins show a slow average rotation with a regression line $V_{\text {rot }}=7.6 \pm 1.4-$ $0.6 \pm 6.0 \mathrm{~km} \mathrm{~s}^{-1} \mathrm{deg}^{-1}$ (Fig. 1a; reflected means in Fig. 1b). The dispersion is $83 \mathrm{~km} \mathrm{~s}^{-1}$, larger than the mean rotational velocity for any bin; there are some retrograde orbits. The velocity gradient of the OH/IR stars is similar to that of planetaries and Miras in the bulge region (Menzies 1990). Minitti et al. (1992) found a similar gradient for two samples of $\mathrm{K}$ giants. These gradients are also similar to those observed in the unresolved bulge light of edge-on galaxies such as NGC 4565 (Kormendy and Illingworth 1982). The rotation in these bulges was interpreted as furnishing a fraction of the total support sufficient to account for the oblateness ratio c/a.

The large difference in the rotation speed of the bulge objects and of the gas clouds that define the Galactic rotation curve at the same longitudes presents no problem if the bulge is related to the halo, as advocated by Carney et al. (1990). These authors note that the very slow average rotation of halo objects at the solar radius $R_{o}$ carries an angular momentum that, after contraction to a tenfold smaller radius in the bulge, is adequate to explain the average rotation of bulge objects.

It seems likely that there is a disk population embedded in the bulge that connects the OH/IR stars near the GC with disk objects outside the bulge. Its members should show rotational velocities, maximum luminosities, and latitude distribution that set them apart from bulge members. Identifying them may require a considerable effort.

\section{REFERENCES}

Blommaert, J.A.D.L., van Langenvelde, H.J., Habing, H. J., van der Veen, W.E.C.J., and Epchtein, N. 1992, Feast Symposium, preprint.

Burton, W. B., and Gordon, M. A. 1978, A\&A, 63, 7.

Carney, B.W., Latham, D.W., and Laird, J.B. 1990, in Bulges of Galaxies, B. Jarvis and D. Terndrup, eds. (Garching: ESO), p. 127.

Catchpole, R.M., Whitelock, P.A., and Glass, I.S., 1990, MNRAS, 247, 479.

Dame, T. M., Ungerechts, H., Cohen, R. S., de Geus, E. J., Grenier, I. A., May, J., Murphy, D. C., Nyman, L.-A., and Thaddeus, P. 1987, ApJ, 322, 706.

Feast, M. W. 1989, in The Gravitational Forces Perpendicular to the Galactic Plane, A. G. Davis, and P. K. Lu eds. (Schenectady: L. Davis Press), p. 210.

Frogel, J. A., and Whitford, A.E., 1987, ApJ, 320, 199.

Kormendy, J., and Illingworth, G. 1982, ApJ, 256, 460.

Lewis, J. R., and Freeman, K. C. 1989, AJ, 97, 139.

te Lintel Hekkert, P., Caswell, J. L., Habing, H. J., Haynes, R. F. and Norris, R. F. 1991, A\&AS, 90, 327.

Lindqvist, M., Winnberg, A., Habing, H. J., and Matthews, H. E. 1992, A\&AS, 92, 43.

Lindqvist, M., Habing, H. J., and Winnberg, A. 1992, A\&A, 259, 118.

Menzies, J. W. 1990, in Bulges of Galaxies, B. Jarvis and D. Terndrup, eds. (Garching: ESO), p. 115.

Minitti, D., White, S. D.M., Olzewski, E. W., and Hill, J. M. 1992, ApJ Lett., 393, L47.

van der Veen, W.E.C.J., and Habing, H.J. 1990, A\&A, 231, 404.

Wielen, R., Dettbarn, C., Fuchs, B. Jahreiss, H., and Radons, G. 1992, in The Stellar Populations of Galaxies, B. Barbuy and A. Renzini, eds., (Dordrecht: Kluwer), p. 81 